Sitientibus Série Ciências Físicas 16, sf01609-1 (2020)

\title{
Evolução Orbital dos Exoplanetas (TRAPPIST-1e e TRAPPIST-1g) que estão na Zona Habitável da Estrela TRAPPIST-1
}

\author{
Orbital Evolution of the Exoplanets (TRAPPIST-1e and TRAPPIST-1g) \\ in the Habitable Zone of the Star TRAPPIST-1
}

\author{
S.R.P. Maciel e J.P.S. Carvalhd* \\ Centro de Ciência e Tecnologia em Energia e Sustentabilidade - UFRB \\ Av. Centenário, 697, Sim. Feira de Santana - BA - 44042-280
}

\begin{abstract}
A abordagem central baseia-se no problema de três corpos, em que é analisada a dinâmica secular de um sistema composto por uma estrela central e dois planetas sob influência gravitacional mútua, considerando a perturbação devido ao efeito da atração gravitacional do terceiro corpo em órbita elíptica e inclinada até a terceira ordem do potencial perturbador. Apresentamos uma análise da evolução orbital dos exoplanetas que estão na zona habitável da estrela. O software Maple é usado para fazer as integrações numéricas, outro software também utilizado é o Universe Sandbox para comparar o resultado obtido das integrações das equações analíticas devido a perturbação do terceiro corpo. Mostramos que os exoplanetas (TRAPPIST-1e e TRAPPIST-1g) que estão na zona habitável da estrela TRAPPIST-1 permanecem dentro da zonal habitável ao longo do tempo.
\end{abstract}

Palavras-chaves: exoplaneta; habitabilidade; evolução orbital.

The central approach is based on the three-body problem, where the secular dynamics of a system composed of a central star and two planets under mutual gravitational influence is analyzed, considering the perturbation due to the effect of the gravitational attraction of the third body in elliptical and inclined orbit up to the third order of the disturbing potential. We present an analysis of the orbital evolution of the exoplanets that are in the habitable zone of the star. The Maple software is used to perform numerical integrations, another software also used is Universe Sandbox to compare the result obtained from integrations of the analytical equations due to the perturbation of the third body. We show that the exoplanets (TRAPPIST-1e and TRAPPIST-1g) that are in the habitable zone of the star TRAPPIST-1 remain within the habitable zone over time.

Keywords: exoplanet; habitability; orbital evolution.

\section{INTRODUÇÃO}

Um exoplaneta é um planeta que está em órbita em torno de outra estrela que não seja o Sol e, portanto, não pertence ao Sistema Solar. Se localizam há anos luz da Terra, dificultando a sua identificação e a aquisição de informações sobre as suas propriedades. A quantidade de exoplanetas confirmados cresceu de forma acelerada com o passar do tempo, em que volumosas descobertas de exoplanetas mostraram uma variedade surpreendente de configurações de sistemas exoplanetários. É possível que di-

*E-mail: jeanfeg@gmail.com versos planetas extra-solares também estejam em zonas habitáveis, situando-se em órbitas em torno das suas estrelas com condições favoráveis ao surgimento da vida. Com isso surgiu a busca por planetas que possam abrigar vida, com características similares as da Terra que estejam na zona temperada do seu sistema planetário. Existe uma grande diversidade de estrelas e planetas que as orbitam. Tal parece ser o caso dos planetas que orbitam a estrela TRAPPIST-1.

Pesquisadores acreditam que dos sete exoplanetas que ela abriga, três orbitam na zona habitável do sistema, para [1] essa descoberta pode ajudar os cientistas a descobrirem mais 
sobre a evolução planetária em estrelas diferentes da nossa. Dessa forma, o interesse no sistema exoplanetário TRAPPIST-1, pode contribuir no entendimento da estabilidade dos exoplanetas na zona habitável, em que vemos o crescente desenvolvimento do conhecimento, um deles ligado aos estudos da Mecânica Celeste, por meio da qual esse trabalho se desenvolveu. Uma motivação desse trabalho são as aplicações da mecânica moderna aos fenômenos celestes e o desenvolvimento de teorias matemáticas fundamentais relacionadas com a Astronomia. Esta área também está relacionada ao estudo da Dinâmica de Sistemas Planetários, abrangendo planetas, satélites e asteroides do Sistema Solar e de sistemas planetários extra-solares, também estudam-se as origens de aspectos observados no Sistema Solar possibilitando determinar as distâncias e as posições dos astros, calcular órbitas de satélites artificiais em torno da Terra, determinar as trajetórias de sondas espaciais enviadas a outros astros do Sistema Solar e determinar as massas de corpos celestes, tais como planetas, satélites, entre outros.

Neste trabalho, analisamos a dinâmica secular de um sistema triplo composto por uma estrela central e dois planetas sob influência gravitacional mútua, que tem como objetivo esclarecer mais aspectos, sobre o comportamento da estabilidade de planetas extra-solares localizados na zona habitável. Considerando as perturbações orbitais devido aos efeitos da força gravitacional no problema de três corpos, para analisar a evolução orbital dos planetas, utilizados para este trabalho somente o TRAPPIST$1 e$ e TRAPPIST- $1 g$.

Investigou-se o comportamento dos parâmetros orbitais, a saber, excentricidade (e), inclinação $(i)$ e o argumento do periastro (g) dos planetas citados, com o objetivo de verificar se os planetas permaneceram na zona habitável ao longo do tempo. Destarte, o resultado desse trabalho é obtido investigando a evolução orbital dos planetas potencialmente habitáveis. Para isto, utilizando-se os parâmetros orbitais citados anteriormente para realizar as simulações numéricas, verificamos se o periastro e o apoastro da órbita podem permanecer na zona habitável ou migrar para outra zona ao longo do tempo devido aos efeitos gravitacionais entre os corpos celestes considerados. Na referência [2] é investigada a dinâmica de um sistema triplo secular de um exoplaneta que se move em torno de uma estrela central perturbada por uma anã marrom. Esse sistema triplo é apresentado como um sistema binário perturbado por uma estrela distante, sendo que a distância entre a estrela e o exoplaneta é muito pequena quando comparada com a distância do terceiro corpo (anã marrom). Com isso, é apresentado um estudo através de formulações de modelos matemáticos e a realização das simulações numéricas de um sistema de equações diferenciais não lineares desenvolvidos no software Maple considerando o sistema planetário real já catalogado.

A estrela TRAPPIST-1, objeto de estudo deste trabalho, abriga sete exoplanetas próximo ao tamanho da Terra que orbitam uma estrela ultrafria [3], os planetas se encontram em órbita da tênue estrela vermelha TRAPPIST- 1 com apenas 8 por cento da massa do nosso Sol, tornando-a uma estrela mais fria e menos luminosa, situada aproximadamente 40 anos-luz de distância da Terra. TRAPPIST1 é uma estrela anã do tipo $\mathrm{M}$ muito tardia, da classe espectral M8V. Tratam-se de estrelas bastante comuns [4, com uma alta taxa de ocorrência de sistemas multi exoplanetários [5, 6] e também de exoplanetas do tamanho da Terra situados na zona habitável [7].

É o primeiro sistema planetário encontrado a transitar uma estrela do tamanho de Júpiter, de massa extremamente baixa. Essa relação planeta-estrela favorável abre a possibilidade de caracterizar minuciosamente os exoplanetas, incluindo sondar suas propriedades atmosféricas, com telescópios atuais e de próximas gerações.

Esse sistema foi descoberto pelo método do trânsito estelar, onde o exoplaneta, visto como um ponto negro que cobre uma pequenina porção do astro luminoso, bloqueia parte da sua luz, ocasionando pequenos decréscimos no brilho da estrela que são materializados na forma de curvas de luz. Esse tipo de ob- 
servação revela o diâmetro relativo do planeta comparado à estrela, bem como seu período orbital em volta dela. Planetas com massas diferentes têm as mesmas órbitas e não há uma maneira direta de os distinguir. No entanto, num sistema com múltiplos planetas, os mais massivos perturbam as órbitas dos outros planetas, o que, por sua vez, afeta o momento em que ocorrem os trânsitos. Esses planetas orbitam tão perto da estrela que seus ciclos or- bitais vão de poucas horas (36 horas para o mais próximo) a poucos dias (20 dias para o mais distante). A referência [8] descreve que eles, por estarem tão próximos uns dos outros, interferem entre si gravitacionalmente, por isso os trânsitos em que passam em frente a sua estrela variam ligeiramente. Estas variações dependem das massas dos planetas, das suas distâncias e de outros parâmetros orbitais.

TABELA I: Dados dos planetas selecionados

\begin{tabular}{|l|c|c|}
\hline \multicolumn{1}{|c|}{ PLANETAS } & TR-1e & TR-1g \\
\hline (a) semieixo maior[ua] & 0,02928285 & 0,0451 \\
\hline$($ i $)$ inclinação & $89,86^{\circ}$ & $89,710^{\circ}$ \\
\hline$(g)$ argumento do periastro & 108,37 & 191,34 \\
\hline$(h)$ longitude do nodo ascendente & $0,1^{\circ}$ & $0,1^{\circ}$ \\
\hline$(e)$ excentricidade & 0,00510 & 0,00208 \\
\hline$(m)$ massa (vezes a massa da terra $\left.\mathbf{m}_{\oplus}\right)$ & $0,62 \star \mathbf{m}_{\oplus}$ & $1,34 \star \mathbf{m}_{\oplus}$ \\
\hline
\end{tabular}

\section{MÉTODOS E MODELO MATEMÁTICO}

O problema de três corpos, ou de forma mais geral o problema de n-corpos, vem sendo estudado por diversos autores [912]. É sabido que o problema de n-corpos possui dez integrais primeiras que permitem reduzir a sua ordem. Essas integrais são as únicas conhecidas [9]. Soluções particulares do problema geral de três corpos foram estabelecidas por Lagrange em 1772. O problema circular restrito de três corpos foi originalmente formulado por Euler em 1772, em que um dos corpos tem massa muito menor que os outros dois ou está muito distante dos outros dois, não influenciando, assim, o movimento dos outros dois corpos, mas é influenciado por estes. Vale destacar as contribuições de Bruns baseado em várias integrais de movimento: momento total, centro de massa, momento angular total e energia total e de Poincaré em que as trajetórias dos corpos são extremamente sensíveis as condições iniciais impostas. Neste trabalho, consideramos o problema de três corpos (os três corpos são massivos) em que o potencial perturbador é desenvolvido até a terceira ordem e substituído nas equações planetárias de Lagrange onde é integrado numericamente para analisar o comportamento orbital de exoplanetas.

Partindo da premissa do problema estelar de três corpos, considerou-se os dados orbitais das Tabelas apresentadas em [3] e 8]. Para a modelagem matemática, utilizamos a abordagem apresentada em [2]. Para integrar numericamente utilizamos o Software Maple, cujos resultados apresentamos nos gráficos de excentricidade $(e)$ versus tempo $(t)$ de cada objeto, utilizando as informações dos objetos listados nas Tabela [1.

Desta forma, analisamos o comportamento orbital dos exoplanetas TR-1e e TR- $1 g$, considerando os elementos orbitais: semieixo maior $(a)$, excentricidade $(e)$, inclinação $(i)$, argumento do periastro $(g)$ e a longitude do nodo ascendente $(h)$. A proposta é realizar simulações numéricas a partir das equações da referência [13] levando em conta a inclinação das órbitas dos planetas. Em [14] é identificado um valor para a zona habitável da es- 
trela hospedeira entre 0,024 e 0,049 unidade astronômica (ua). Consideramos esse valor da zona habitável para analisar a posição do periastro e a excentricidade do exoplaneta TR-1e, o qual está mais próximo da zona quente, para observar se haveria algum tipo de comportamento de migração da zona temperada para a zona quente. Assim, é possível analisar o comportamento orbital dos exoplanetas que orbitam a zona habitável dessa estrela.

Para melhor visualizarmos como se comporta o efeito da excentricidade das órbitas de um exoplaneta sobre o conceito de zona habitável, utilizamos alguns valores iniciais para a excentricidade como mostrado nas Figuras 3,6, para poder identificar suas alterações levando em conta o efeito gravitacional do terceiro corpo. Como os valores das excentricidades não são bem determinados, apresentamos as simulações numéricas para um intervalo de valores iniciais de excentricidades tomando os valores da Tabela 3 nas referências [3, 8].

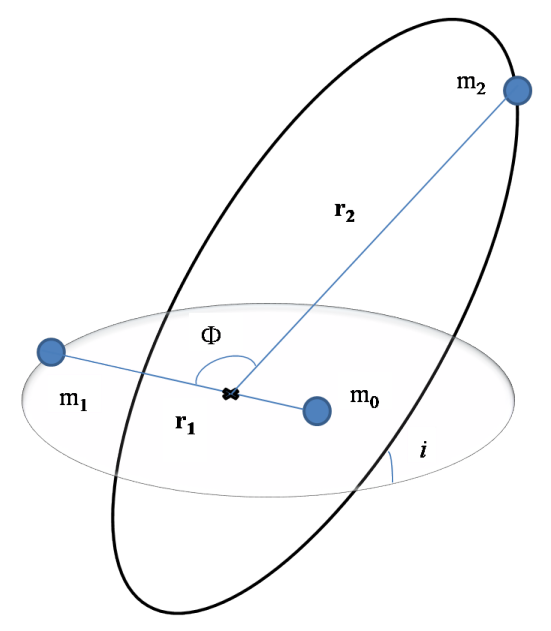

FIGURA 1: Sistema de coordenadas usado para descrever o problema de três corpos. Fonte: referência 2].

\section{A. Equações de Movimento}

A modelagem das equações de movimento é apresentada levando em conta o problema de três corpos elíptico e inclinado [12]. Para as perturbações orbitais, em [13] esse potencial perturbador é expandido até a quinta ordem, mas neste caso consideramos apenas até a terceira ordem como é apresentado em [15, considerando a inclinação do corpo perturbador. O potencial perturbador é desenvolvido em forma fechada até a terceira ordem em um pequeno parâmetro da razão de semieixos $\left(\alpha=a_{1} / a_{2}\right)$ em que $a_{1}$ e $a_{2}$ são os semieixos maior dos planetas TR-1e e TR-1g, respectivamente.

O sistema triplo em estudo é caracterizado por um planeta $m_{1}$ em uma órbita interna elíptica em torno do centro de massa do sistema $m_{0}-m_{1}$, orbitando uma estrela central $m_{0}$, também se movendo em torno do centro de massa do sistema $m_{0}-m_{1}$ e mais um perturbador (o planeta $m_{2}$ ) movendo-se em uma órbita elíptica externa ao redor do centro de massa do sistema. O hamiltoniano do sistema triplo pode ser escrito [16] como segue,

$$
\begin{aligned}
F & =\frac{G m_{0} m_{1}}{2 a_{1}}+\frac{G\left(m_{0}+m_{1}\right) m_{2}}{2 a_{2}} \\
& +\frac{G}{a_{2}} \sum_{j=2}^{\infty} \alpha^{j} M_{j}\left(\frac{r_{1}}{a_{1}}\right)^{j}\left(\frac{a_{2}}{r_{2}}\right)^{j+1} P_{j}(\cos \Phi),
\end{aligned}
$$

$\mathrm{O}$ vetor $\mathbf{r}_{1}$ representa a posição de $m_{1} \mathrm{em}$ relação a $m_{0}$ e o vetor $\mathbf{r}_{2}$ é a posição do corpo $m_{2}$ em relação ao centro de massa da órbita interna (veja a Figura 1). $\Phi$ é o ângulo entre $\mathbf{r}_{1}$ e $\mathbf{r}_{2}$ e $G$ é a constante gravitacional, $P_{j}$ são os polinômios de Legendre, assim,

$$
M_{j}=m_{0} m_{1} m_{2} \frac{m_{0}^{j-1}-\left(-m_{1}\right)^{j-1}}{\left(m_{0}+m_{1}\right)^{j}} .
$$

Desenvolvemos o potencial perturbador levando em consideração a expressão para $\cos \Phi$ na Eq.(3) escrita conforme [17], com $s_{1}=$ sen $i_{1}, c_{1}=\cos i_{1}, s_{2}=\operatorname{sen} i_{2}$, e $c_{2}=\cos i_{2}$. Aqui $i_{j}, g_{j}, h_{j}$ e $f_{j}$ (para $j=1,2$ ) são a inclinação, argumento do periastro, longitude do nodo ascendente e a anomalia verdadeira da órbita interna e externa, respectivamente, 


$$
\begin{aligned}
\cos (\Phi) & =\frac{1}{4}\left(-1+c_{2}\right)\left(-1+c_{1}\right) \cos \left(f_{1}+g_{1}-h_{1}-f_{2}-g_{2}+h_{2}\right)+\frac{1}{4}\left(1+c_{1}\right)\left(1+c_{2}\right) \times \\
& \times \cos \left[f_{1}+g_{1}+h_{1}-f_{2}-g_{2}-h_{2}\right]-\frac{1}{4}\left(1+c_{2}\right)\left(-1+c_{1}\right) \cos \left(f_{1}+g_{1}-h_{1}+f_{2}+g_{2}+h_{2}\right) \\
& -\frac{1}{4}\left(-1+c_{2}\right)\left(1+c_{1}\right) \cos \left(f_{1}+g_{1}+h_{1}+f_{2}+g_{2}-h_{2}\right) \\
& +\frac{1}{2} s_{1} s_{2}\left[\cos \left(f_{1}+g_{1}-f_{2}-g_{2}\right)-\cos \left(f_{1}+g_{1}+f_{2}+g_{2}\right)\right] \\
R_{2 C}= & -\frac{45}{2} \frac{\beta_{3} L_{1}^{4}}{L_{2}{ }^{6}\left(1-e_{2}^{2}\right)^{3 / 2}} 1 / 6 e_{1}^{2}\left(c_{2}-1\right)\left(c_{2}+1\right)\left(c_{1}+1\right)^{2} \cos \left(2 g_{1}-2 h_{2}+2 h_{1}\right) \\
+ & \frac{1}{6} e_{1}^{2}\left(c_{2}-1\right)\left(c_{2}+1\right)\left(c_{1}-1\right)^{2} \cos \left(2 g_{1}+2 h_{2}-2 h_{1}\right)+2 / 3 c_{2} s_{1} s_{2} e_{1}^{2}\left(c_{1}-1\right) \cos \left(2 g_{1}-h_{1}+h_{2}\right) \\
+ & \frac{2}{3} c_{2} s_{1} s_{2} e_{1}^{2}\left(c_{1}+1\right) \cos \left(2 g_{1}+h_{1}-h_{2}\right)-1 / 5\left(c_{2}+1\right)\left(c_{1}-1\right)\left(c_{1}+1\right)\left(c_{2}-1\right)\left(2 / 3+e_{1}^{2}\right) \times \\
& \times \cos \left(-2 h_{2}+2 h_{1}\right)-4 / 5 c_{1} s_{2} s_{1}\left(2 / 3+e_{1}^{2}\right) c_{2} \cos \left(h_{1}-h_{2}\right) \\
+ & \left(-1 / 3+c_{2}^{2}\right)\left(\left(c_{1}^{2}-1\right) e_{1}^{2} \cos \left(2 g_{1}\right)-3 / 5\left(c_{1}^{2}-1 / 3\right)\left(2 / 3+e_{1}^{2}\right)\right) .
\end{aligned}
$$

Para o modelo considerado no presente trabalho, é necessário calcular os termos $R_{2}$ e $R_{3}$ da função perturbadora devido aos termos $P_{2}$ e $P_{3}$, respectivamente. Temos então,

$$
\begin{aligned}
& R_{2}=\frac{G}{a_{2}} \alpha^{2} M_{2}\left(\frac{r_{1}}{a_{1}}\right)^{2}\left(\frac{a_{2}}{r_{2}}\right)^{3} P_{2}(\cos \Phi), \\
& R_{3}=\frac{G}{a_{2}} \alpha^{3} M_{3}\left(\frac{r_{1}}{a_{1}}\right)^{3}\left(\frac{a_{2}}{r_{2}}\right)^{4} P_{3}(\cos \Phi) .
\end{aligned}
$$

O potencial perturbador dado pela Eq.(1) é,

$$
F=R_{0}+R_{2}+R_{3},
$$

em que $R_{0}$ é,

$$
R_{0}=\frac{G m_{0} m_{1}}{2 a_{1}}+\frac{G\left(m_{0}+m_{1}\right) m_{2}}{2 a_{2}} .
$$

O desenvolvimento do potencial perturbador com o objetivo de reescrita em função dos elementos orbitais encontra-se em [15]. Após manipulações algébricas usando equações conhecidas da Mecânica Celeste e da aplicação do método da dupla média, a equação do termo de segunda ordem torna-se a Eq.(4) com as funções $L_{1}, L_{2}, \beta_{3}$, e $\beta_{4}$ dadas por [15] :

$$
\begin{aligned}
L_{1} & =\frac{m_{0} m_{1} \sqrt{G\left(m_{0}+m_{1}\right) a_{1}}}{m_{0}+m_{1}}, \\
L_{2} & =\frac{m_{2}\left(m_{0}+m_{1}\right)}{m_{0}+m_{1}+m_{2}} \times \\
& \times \sqrt{G\left(m_{0}+m_{1}+m_{2}\right) a_{2}}, \\
\beta_{3} & =\frac{1}{16} \frac{G^{2}\left(m_{0}+m_{1}\right)^{7} m_{2}^{7}}{\left(m_{0}+m_{1}+m_{2}\right)^{3} m_{0}^{3} m_{1}^{3}}, \\
\beta_{4} & =\frac{1}{4} \frac{G^{2}\left(m_{0}+m_{1}\right)^{9} m_{2}^{9}\left(m_{0}-m_{1}\right)}{\left(m_{0}+m_{1}+m_{2}\right)^{4} m_{0}^{5} m_{1}^{5}} .
\end{aligned}
$$

O potencial devido ao termo $R_{3}$ não será apresentado aqui porque é muito longo. Depende dos termos $g_{1}, g_{2}, h_{1}$ e $h_{2}$ (quatro graus de liberdade). $\mathrm{O}$ termo $R_{3}$ é desenvolvido usando a Eq.(6) e $\cos (\Phi)$ é substituído pela Eq.(3).

$$
R_{3 C}=\frac{2 \beta_{4} L_{1}^{6} a_{2}^{4} r_{1}^{3}}{L_{2}^{8} r_{2}^{4} a_{1}^{3}}\left[5 \cos ^{3}(\Phi)-3 \cos (\Phi)\right]
$$

Nosso potencial perturbador pode ser reescrito,

$$
R=R_{2 C}+R_{3 C},
$$


A Eq. (14) é substituída nas Equações Planetárias de Lagrange para analisarmos as influências causadas pelas perturbações consideradas na dinâmica do exoplaneta. As simulações numéricas são realizadas usando o Software Maple via Equações Planetárias de Lagrange, um sistema de equações diferenciais não lineares descritas pelas equações:

$$
\begin{aligned}
\frac{d e}{d t} & =\frac{-\sqrt{1-e^{2}}}{n a^{2} e} \frac{\partial R}{\partial g}+\frac{1-e^{2}}{n a^{2} e} \frac{\partial R}{\partial M} \\
\frac{d i}{d t} & =\frac{-1}{n a^{2} \sqrt{1-e^{2} \operatorname{sen} i}} \frac{\partial R}{\partial h} \\
& +\frac{\cos i}{n a^{2} \sqrt{1-e^{2} \operatorname{sen} i}} \frac{\partial R}{\partial g} \\
\frac{d h}{d t} & =\frac{1}{n a^{2} \sqrt{1-e^{2} \operatorname{sen} i}} \frac{\partial R}{\partial i} \\
\frac{d g}{d t} & =\frac{\sqrt{1-e^{2}} \frac{\partial R}{n a^{2} e}-\frac{\cos i}{n a^{2} \sqrt{1-e^{2} \operatorname{sen} i}}}{\partial i}
\end{aligned}
$$

em que $R$ é a função perturbadora dada pela Eq.(14). As equações de movimento são desenvolvidos de forma fechada para evitar expansões em séries de potência da excentricidade e inclinação. Para eliminar os termos de curto período do potencial perturbador o método da média dupla é aplicado.

\section{B. Materiais}

O desenvolvimento deste trabalho utilizou das ferramentas de simulações numéricas como o Software Maple e o programa Universe Sandbox, na criação de representações das orbitais dos planetas estudados, para o qual se fez necessário utilizar os dados reelativos aos exoplanetas que orbitam a estrela TRAPPIST-1.

Recorrendo ao programa Universe Sandbox, criou-se simulações para exibir o comportamento desse sistema, testando diferentes valores de excentricidade do exoplaneta TRAPPIST-1e e o TRAPPIST- $1 g$ para ver em quais condições eles podem ser considerado um planeta habitável e se esses poderão mudar suas órbitas da zona habitável para a zona quente ou fria devido as influências dos outros corpos. A Figura 2 é uma representação do sistema TRAPPIST-1, criada pelo autor por meio do programa Universe Sandbox, para mostrar a posição de cada exoplaneta no sistema em questão. Como as excentricidades desses planetas ainda não são bem definidas, as simulações ajudam a interpretar a dinâmica variando os valores iniciais das excentricidades.

\section{RESULTADOS E DISCUSSÕES}

O potencial perturbador devido ao terceiro corpo é substituído nas equações planetárias de Lagrange e integrado numericamente via software Maple. As Figuras a seguir mostram o resultado do comportamento da excentricidade, inclinação e argumento do periastro em relação ao tempo do sistema de exoplanetas da estrela TRAPPIST (TR-1) em que os exoplanetas TR$1 e$ e TR-1g são mutuamente perturbados. Note que os gráficos da posição do periastro são apenas para o exoplaneta TR-1e.

\section{A. Resultados}

Como as excentricidades desses planetas ainda não são determinadas com precisão, as Figuras 3 6 6 são simuladas para diferentes valores da excentricidade inicial (ver a legenda das figuras). A Figura 3 mostra que a excentricidade e a inclinação do planeta TR-1e são mais perturbados do que o planeta TR- $1 g$, isso se deve pelo fato do TR- $1 g$ ser mais massivo. Note também que a inclinação varia muito pouco considerando à atração gravitacional mútua, neste caso, não há um flip da inclinação (mudar de direta para retrograda), mesmo considerando o termo de ordem 3 do potencial perturbador. Já em [2], [13, [15, acontece o flip, pois o corpo perturbador é uma anã marrom, o que causa uma maior perturbação na órbita do planeta. Como no caso estudado neste trabalho o perturbador é um outro planeta com massa ligeiramente maior, então o efeito gravitacional não é capaz de causar o flip. Ainda nesta figura, podemos verificar que a posição do periastro e do apoastro varia muito pouco 
para o par de valores de excentricidade considerados. Destacando que a zona habitável da estrela é a região entre as curvas verde e vermelha no gráfico da posição do periastro e do apoastro.

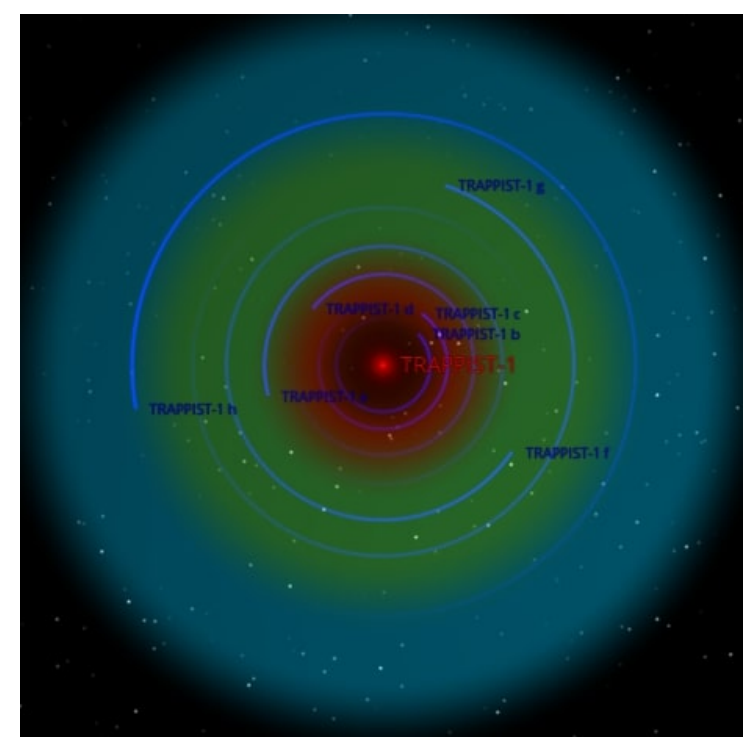

FIGURA 2: Sistema TRAPPIST-1 e seus 7 planetas orbitando ao redor da estrela. A cor azul representa a zona fria, a cor verde representa a zona morna (zona habitável) e vermelha representa a zona quente. (Universe Sandbox).

As Figuras 4, 5 e 6 mostram que aumentando o valor inicial da excentricidade aumenta a variação da excentricidade e da variação do periastro e apoastro, quanto mais elípitca forem as órbitas mais perturbados são os planetas. Como podemos ver na Figura 6 no gráfico da posição do periastro, a órbita do exoplaneta TR-1e apresenta grande amplitude de variação quando comparada com os outros casos analisados, e assim, o exoplaneta TR-1e se aproxima da zona quente. Já a inclinação não houve diferença quando mudamos o valor inicial da excentricidade. Tudo indica que seria necessário um corpo mais massivo para perturbar a inclinação orbital dos planetas. Em todas as simulações o planeta TR-1e permaneceu dentro da zona habitável ao longo do tempo.

\section{B. Discussões}

Para melhor analisarmos como se comporta o efeito da excentricidade das órbitas de um exoplaneta sobre o conceito de zona habitável, utilizamos alguns valores para a excentricidade como mostrado nas Figuras expostas anteriormente. Para melhor visualizar suas alterações são utilizados pares de excentricidades proporcionais aos valores iniciais proposto por [8]. Vemos que apesar de ser perturbado por outros corpos do sistema e das alterações em suas excentricidades, o mesmo continuará orbitando na zona habitável da estrela. Mostramos os gráficos da posição do periastro $\left(\mathbf{r}_{\mathbf{p}}\right)$ e do apoastro $\left(\mathbf{r}_{\mathbf{a}}\right)$ em relação ao tempo apenas para o TR-1e. A escolha é devida ao fato do TR$1 g$ ser mais massivo, e assim perturba mais a órbita do TR-1e.

Ao analisarmos os resultados de excentricidade e localização do periastro e apoastro em relação ao tempo dos exoplanetas TR-1e, e TR$1 g$ a excentricidade do exoplaneta em relação ao tempo nas Figuras 3, 4, 5 e 6. Note que o planeta TR-1 $g$ é o que mais perturba a excentricidade do planeta TR-1e, e há uma variação de amplitude que descreve o efeito da atração gravitacional entre os dois corpos, uma das explicações seria devido ele ser um dos planetas mais massivo do sistema, o que acaba causando uma atração gravitacional sobre o TRAPPIST-1e. No entanto, vemos que apesar de ser perturbado por outro corpo do sistema, o TRAPPIST-1e (ver Figura 6) continua orbitando na zona habitável da estrela. Na Figura 6 entre as retas vermelha (borda da zona quente) e verde (borda da zona fria) que representa a zona habitável para o sistema estudado. Como se pode ver nesta figura, o apoastro e o periastro continuam dentro da zona habitável ao longo do tempo.

Como mostram as Figuras de 3 até 6 . quando menor o valor inicial da excentricidade, menor é a variação dos parâmetros orbitais. Agora aumentando o valor inicial da excentricidade, as amplitudes de variação da excentricidade aumentam. Para a inclinação, não houve praticamente alteração nos casos analisados. Tudo indica que seria necessário um 
corpo mais massivo para perturbar a inclinação orbital dos planetas. Lembrando que a zona habitável da estrela é a região entre as curvas verde e vermelha. Em todas as simulações o planeta permaneceu dentro da zona habitável ao longo do tempo.
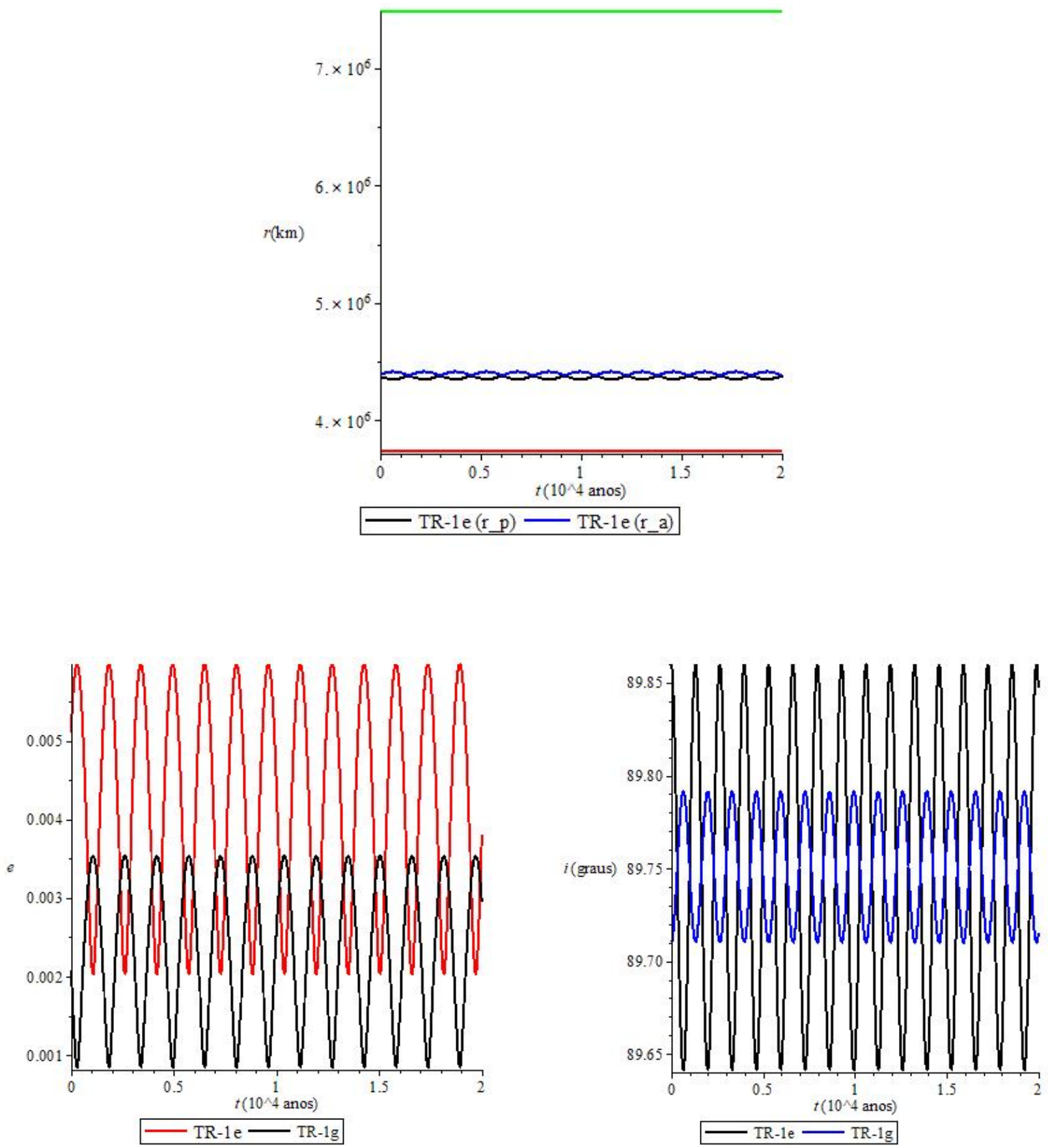

FIGURA 3: A partir do topo temos os gráficos de Zona, Excentricidade e Inclinação, respectivamente, para TR-1g $(e=0,00208)$, TR-1e $(e=0,00510)$. A zona habitável da estrela é a região entre as curvas verde e vermelha no gráfico da posição do periastro e do apoastro. Fonte: figuras elaboradas pelo autor. 
A maior variação dentro da zona habitável foi para o caso com maior valor inicial da excentricidade como mostra a Figura 6, em que o planeta se aproximou da zona quente. Di- ante dessas simulações os resultados mostram que as órbitas desses planetas são estáveis, considerando apenas a interação mútua dos planetas TR-1e e TR-1g.
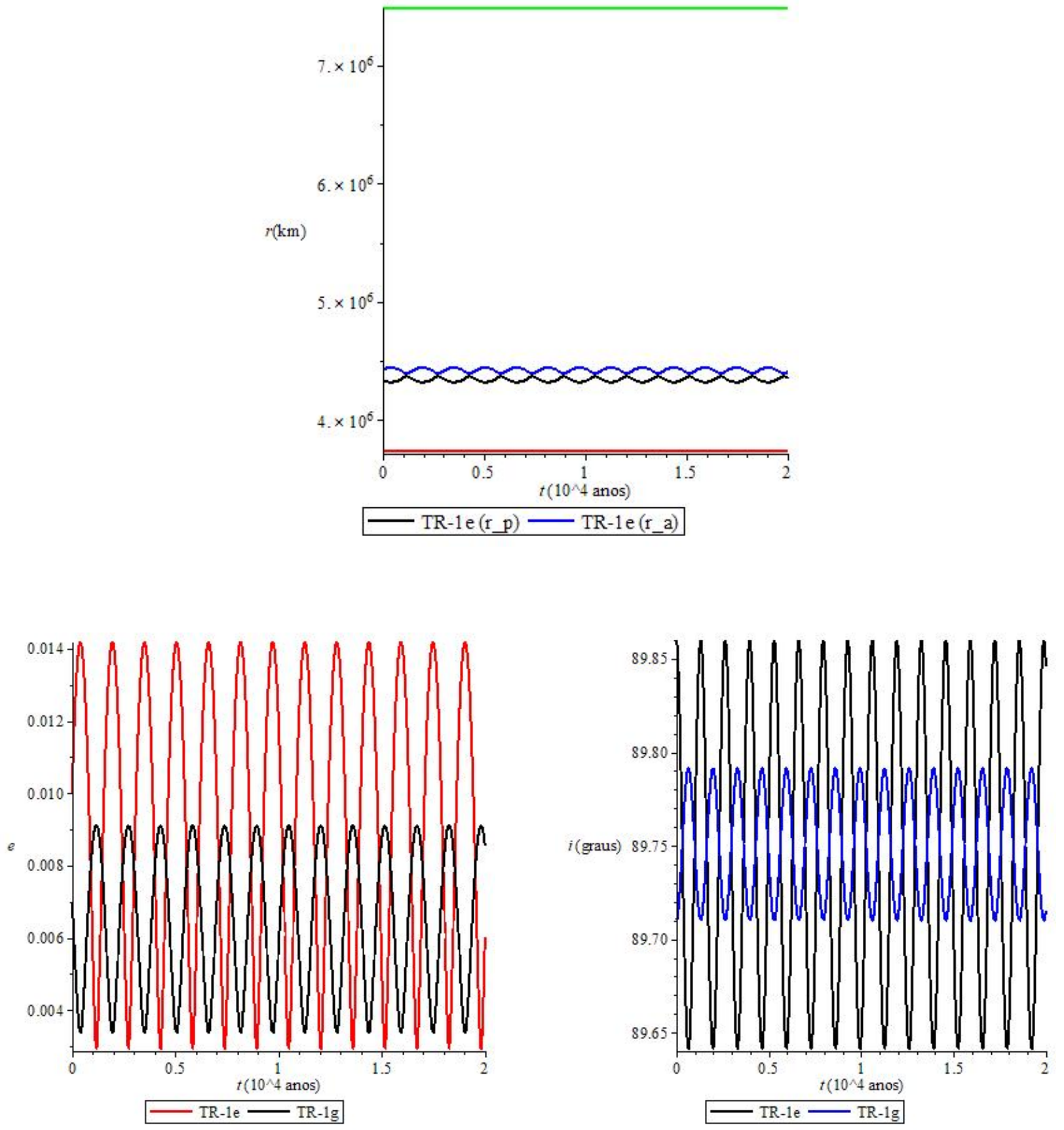

FIGURA 4: A partir do topo temos os gráficos de Zona, Excentricidade e Inclinação, respectivamente, para TR-1g $(e=0,007)$; TR-1e $(e=0,01)$. A zona habitável da estrela é a região entre as curvas verde e vermelha no gráfico da posição do periastro e do apoastro. Fonte: figuras elaboradas pelo autor. 

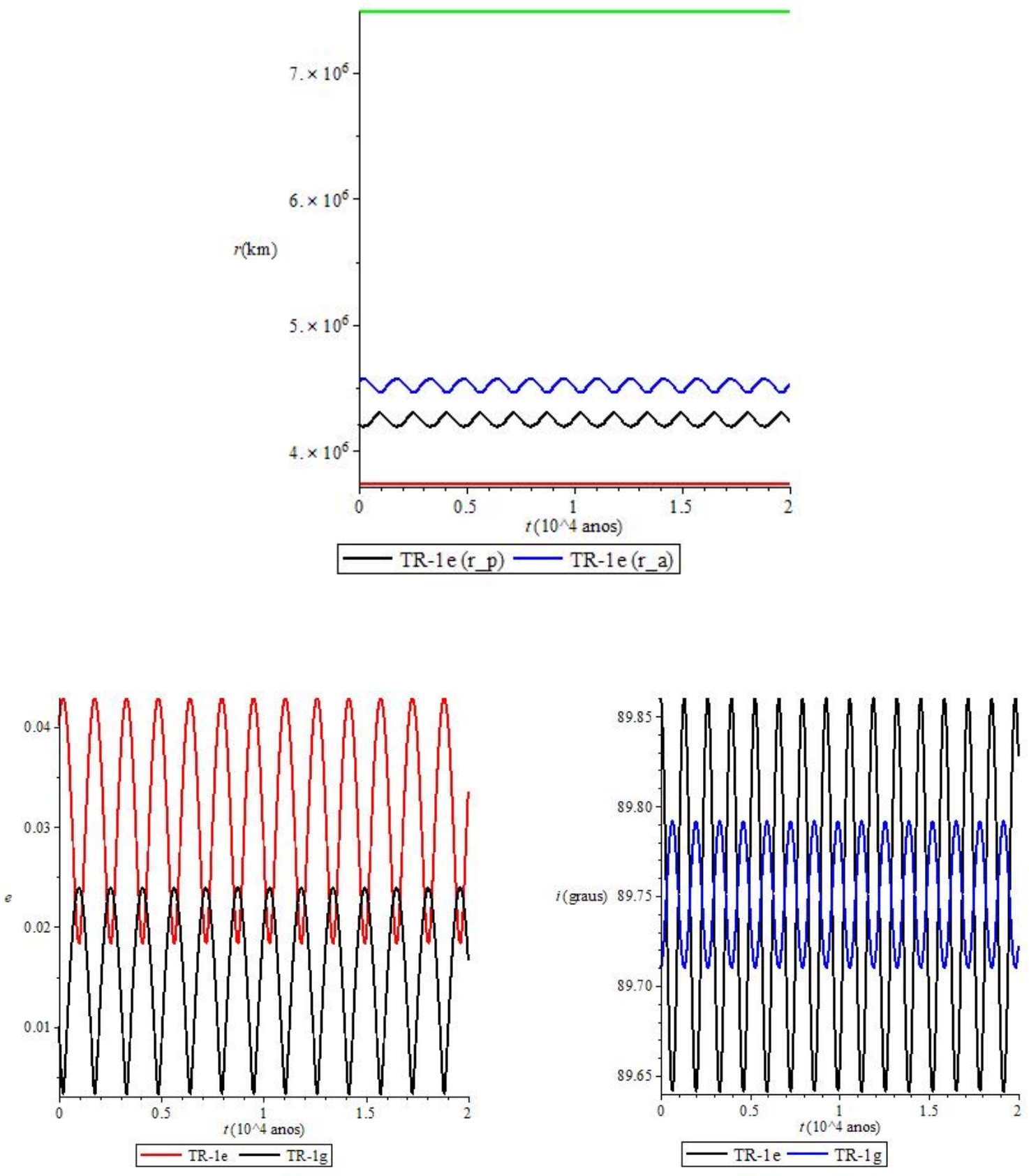

FIGURA 5: A partir do topo temos os gráficos de Zona, Excentricidade e Inclinação, respectivamente, para TR-1g $(e=0,01)$; TR-1e $(e=0,04)$. Fonte: figuras elaboradas pelo autor. A zona habitável da estrela é a região entre as curvas verde e vermelha no gráfico da posição do periastro e do apoastro.

A Figura 7 ilustra as órbitas dos planetas TR-1e, TR-1 $g$ em torno da estrela. A Figura 8 mostra por meio da simulação no programa Universe Sandbox como a excentricidade do e- xoplaneta pode interferir em sua órbita dentro da zona habitável. Neste caso, o apoastro da órbita sai da zona habitável e se aproxima da zona quente. 

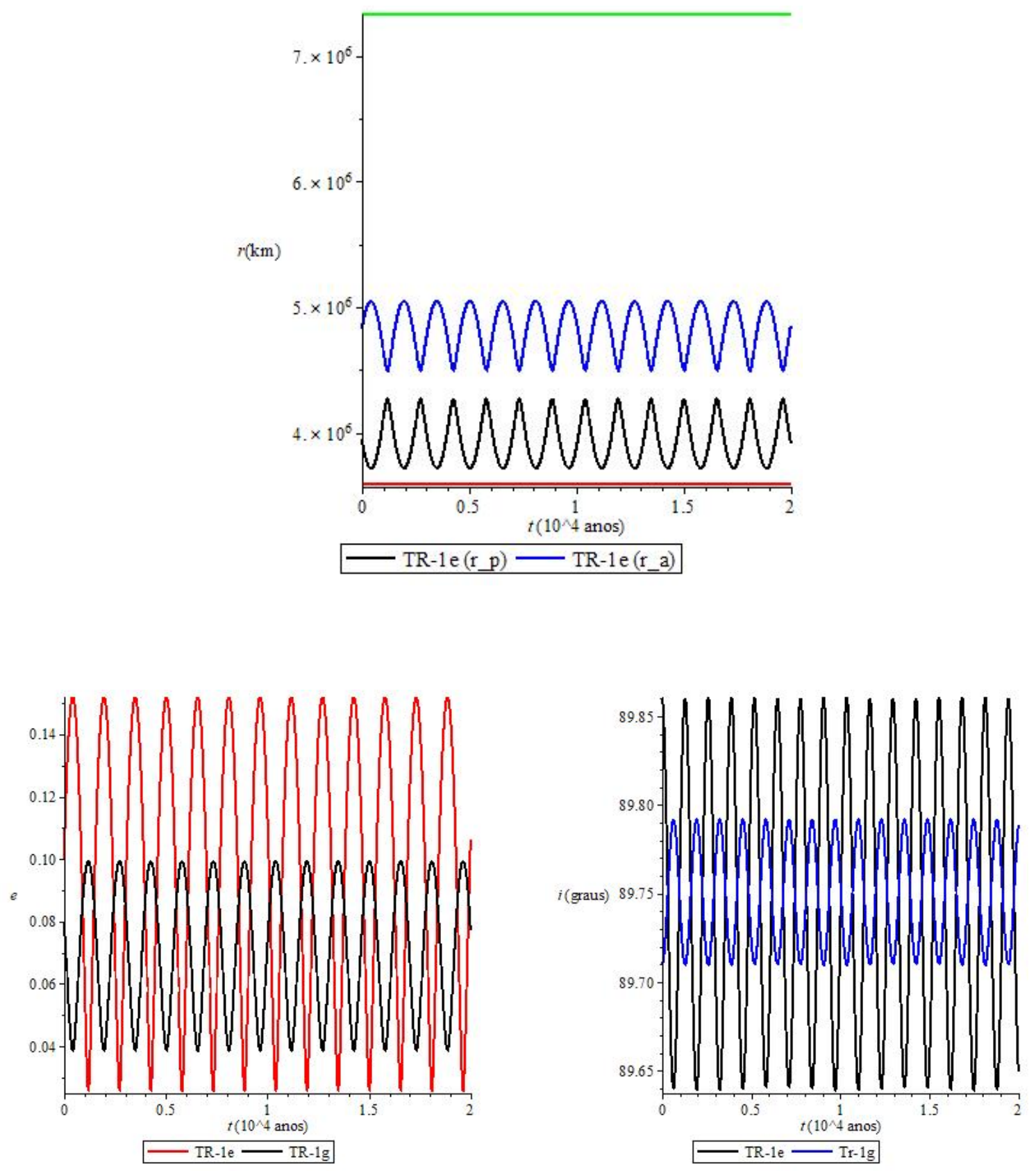

FIGURA 6: A partir do topo temos os gráficos de Zona, Excentricidade e Inclinação, respectivamente, para TR-1g $(e=0,08)$; TR-1e $(e=0,1)$. Fonte: figuras elaboradas pelo autor. A zona habitável da estrela é a região entre as curvas verde e vermelha no gráfico da posição do periastro e do apoastro.

Devido a isso, observa-se que a depender do valor da excentricidade do planeta ele pode fazer com que o planeta migre da zona habitável para outra zona próxima. Como as excentricidades desses planetas ainda não são bem definidas, as simulações ajudam a interpretar a dinâmica variando os valores inici- ais das excentricidades. Note que o periastro do planeta TR-1e se aproxima da zona quente para os valores das excentricidades de (TR-1e) $e=0,1$ e (TR- $1 g) e=0,08$. Vale ressaltar que neste trabalho não apresentamos estudos estatísticos para quantificar os erros associados nos valores iniciais atribuídos para a excentrici- 
dade nas estimativas dos parâmetros orbitais, variações, dentro do fator de correlação existente.

O objetivo inicial foi o de verificar o comportamento da excentricidade, posição do periastro e inclinação dos exoplanetas a partir dos dados disponíveis na literatura. Mas é de suma importância uma nova abordagem para tratar os erros associados para fundamentar matematicamente os procedimentos apresentados para estimar o erro de soluções numéricas.

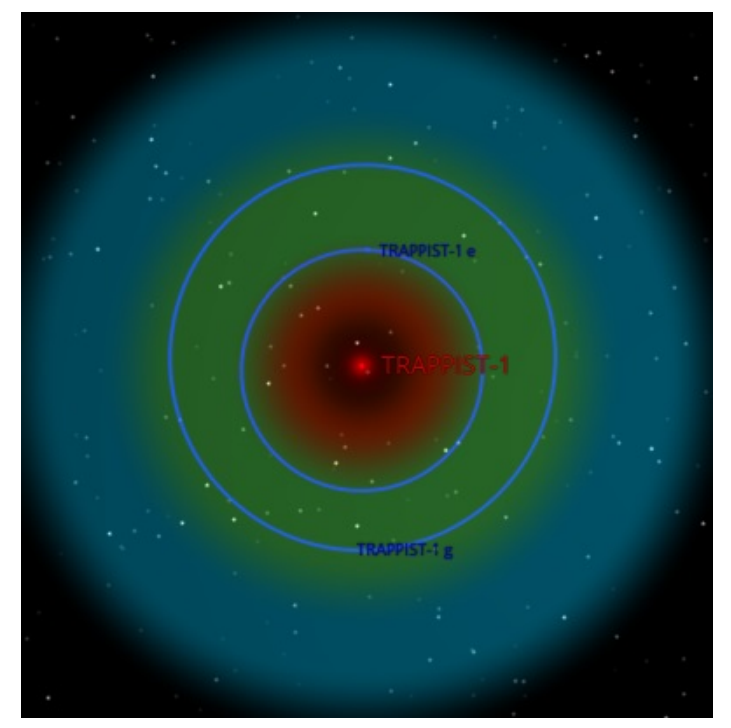

FIGURA 7: Excentricidade inicial: TR-1g $(e=$ $0,0208)$; TR-1e $(e=0,00510)$. Fonte: figura elaborada pelo autor. A cor azul representa a zona fria, a cor verde representa a zona morna (zona habitável) e vermelha representa a zona quente.

\section{CONCLUSÕES}

Nesse estudo consideramos o problema de três corpos, em especial a interação dos exoplanetas que orbitam dentro da zona habitável da estrela mãe, sendo o foco analisar o comportamento das excentricidades do TR-1e e do TR$1 g$, perturbado por seus efeitos gravitacionais. Considerou-se as equações dadas por [13] em que é levado em conta a inclinação do corpo perturbador, ou seja, a inclinação de cada exoplaneta do sistema estudado. Com isso, foi possível também plotar os gráficos com o comportamento da inclinação de cada exoplaneta aqui exposto. O que se observa é que apesar das variações, no geral, esse sistema permanece orbitando dentro da zona habitável da estrela, isso quando o valor máximo aqui utilizado foi de 0,1 para a excentricidade do TR- $1 e$, para valores maiores de excentricidade esses planetas poderão migrar da zona habitável para outra zona próxima, seja a zona quente ou fria. Vale ressaltar também, que os dados aqui utilizados e disponibilizados por alguns autores, não é um valor real já catalogado dos exoplanetas, são valores encontrados a partir de estudos sobre os sistemas, para valores como o da excentricidade, por exemplo, ainda não se tem um valor real fixo ou determinado.

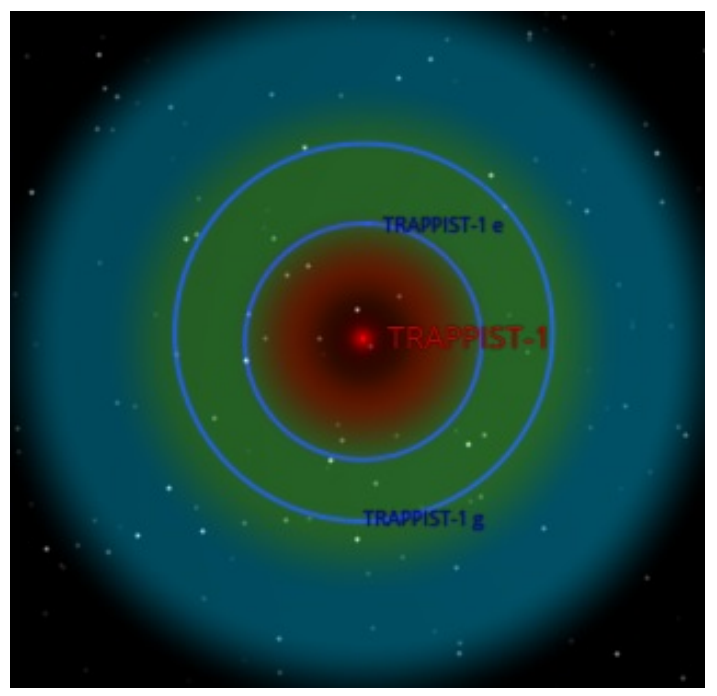

FIGURA 8: Excentricidade final: TR-1g ( $e=$ $0,08)$; TR-1e $(e=0,1)$. Fonte: figura elaborada pelo autor. A cor azul representa a zona fria, a cor verde representa a zona morna (zona habitável) e vermelha representa a zona quente.

\section{Agradecimentos}

Os autores agradecem ao $\mathrm{CNPq}$ pelos contratos 307724/2017 -4, 420674/2016 -0 e ao PIBIC da UFRB. Este trabalho foi patrocinado pelo CNPq. 
[1] A.P. Lincowski, V.S. Meadows, D. Crisp, T.D. Robinson, R. Luger, J.L. Yaeger, G.N. Arney, Evolved Climates and Observational Discriminants for the TRAPPISTg-1 Planetary System. The Astrophysical Journal 867, (1) 76 (2018).

[2] J.P.S. Carvalho, R.V. Moraes, A.F.B.A. Prado, O.C. Winter, Analysis of the secular problem for triple star systems. Journal of Physics: Conference Series 465, (1) (2013).

[3] M. Gillion, A.H.M.J. Triaud, B.-O. Demory, E. Jehin, E. Agol, NASA, University of Bern, STAR Institute. Trappist-1 (2017).

[4] T.J. Henry, W.-C. Jao, J.P. Subasavage, et al.. Astronomical Journal 132, 2360 (2006).

[5] S. Ballard, J.A. Johnson. Astrophysical Journal 816, 66 (2016).

[6] M. Gillion, A.H.M.J. Triaud, B.-O. Demory, et al.. Nature 542, 456 (2017).

[7] C.D. Dressing, D. Charbonneau. Astrophysical Journal 807, 45 (2015).

[8] S.L. Grimm, B.O. Demory, M. Gillon, et al., The nature of the TRAPPIST-1 exoplanets. Astronomy and Astrophysics 613, (2018).

[9] S.S. Fernandes, M.C.F.P.S. Zanardi, Fundamentos de Astronáutica e suas Aplicações. vol.2. São Paulo: EdUFABC (2018).

[10] V.I. Arnold, V.V. Kozlov, A.I. Neishtadt, Mathematical Aspects of Classsical and Celestial Mechanics. Berlin: Springer-Verlag
(2006).

[11] A.F.B.A. Prado, Trajetórias Espaciais e Manobras Assistidas por Gravidade. São José dos Campos: INPE (2001).

[12] M. Valtonen, H. Karttunen, The Three-Body Problem. Cambridge: Cambridge University Press (2006).

[13] J.P.S. Carvalho, R.V. De Moraes, A.F.B.A Prado, D.C. Mourão, O.C. Winter, Analysis of the orbital evolution of exoplanets. Computational \& Applied Mathematics 35, 847 (2016).

[14] M. Gillon, E. Jehin, S.M. Lederer, L. Delrez, J. Wit, A. Burdanov, V.V. Grootel, A.J. Burgasser, A.H.M.J. Triaud, C. Opitom, B.O. Demory, D.K. Sahu, D.B. Gagliun, P. Magain, D. Queloz, Temperate Earth-sized planets transiting a nearby ultracool dwarf star. Nature $\mathbf{5 3 3}$, 221 (2016).

[15] J.P.S. Carvalho, D.C. Mourão, R.V. Moraes, A.F.B.A. Prado, O.C. Winter, Exoplanets in binary star systems: on the switch from prograde to retrograde orbits. Celestial Mechanics and Dynamical Astronomy 124, (1) 73 (2016).

[16] R.S. Harrington, Dynamical evolution of triple stars. The Astronomical Journal 73, (3) 190 (1968).

[17] T. Yokoyama, Possible effects of secular resonances in phobos and triton. Planetary and Space Science 50, (1) 63 (2002). 